\title{
Ontology-based smart learning environment for teaching word problems in mathematics
}

\author{
Aparna Lalingkar • Chandrashekar Ramnathan • \\ Srinivasan Ramani
}

Received: 30 August 2014/Revised: 6 November 2014/Accepted: 6 November 2014/

Published online: 18 November 2014

(C) Beijing Normal University 2014

\begin{abstract}
This paper demonstrates how to use MONTO-machine-readable ontology for teaching word problems in mathematics. MONTO is a combination of various ontologies. After presenting a review of the literature of problem solving and math education, this paper presents a framework that is used for evaluation of the systems available in the literature for teaching problem solving in mathematics. This paper analyzes the existing systems and evaluates them against the framework to state the gaps. It also describes MONTO and demonstrates its use. This paper also discusses how the use of MONTO bridges the gaps found in the literature of tutoring systems used for teaching problem solving. A description of the system implemented using MONTO is included along with the key functionalities of the system derived from the ontology. A discussion of how these functionalities are useful for teaching word problems is included too. This paper also presents the results of a small-scale study conducted to evaluate the functionalities that are derived from the ontology.
\end{abstract}

\section{Introduction and background}

A student who has been taught mathematical problem solving can be strong in analyzing a large amount of quantitative data, can use mathematics in practical ways, and can be analytical both in thinking for herself and in examining the arguments put

\footnotetext{
A. Lalingkar $(\bowtie) \cdot$ C. Ramnathan $\cdot$ S. Ramani International Institute of Information Technology, Bangalore, Bangalore, India e-mail: aparna.1@iiitb.ac.in

C. Ramnathan

e-mail: rc@iiitb.ac.in

S. Ramani

e-mail: ramani.srini@gmail.com
} 
forward by others (Schoenfeld 1992). Good conceptual knowledge and the ability to solve various problems in high school level mathematics form a basis for quality higher education. Hence the study of how Information and Communication Technology has been used for teaching mathematical problem solving at the high school level is important. By the term mathematical problems we mean problems which are solved using mathematical formulas, mathematical logic, and rules. There are various types of problems, but the real training in problem solving begins when word (story) problems get introduced, where students need to process the real world scenario and connect it to mathematical knowledge. Hence, we focused on word (story) problems. While describing his research on teaching mathematical problem solving, Schoenfeld (1985) gave a framework for analysis of mathematical behavior. The framework comprises four components: (a) resources i.e., basic concepts and mathematical knowledge (cognitive aspect); (b) heuristics i.e., broad range of problem-solving strategies illustrated by Polya (1946) and some sub-strategies added by Schoenfeld himself (meta-cognitive aspect); (c) control i.e., efficiency in combining resources and heuristics to take the decision to solve a problem (decision making which is part of meta-cognitive aspect); and (d) belief systems i.e., one's opinion and belief about mathematics and mathematical problem solving (motivational aspect). Despite more than seven decades of work in teaching problem solving (Polya 1946; Newell and Simon 1972; Schoenfeld 1985; Silver 1985; Marshall 1995; Jonassen 2011), classroom teaching of the solving of mathematical problems at the school level has remained a great challenge. In this paper, we initially offer the state of the art of the systems used for teaching word problems in mathematics followed by evaluation of the systems against a rubric developed by extensive review of the literature of mathematical thinking and understanding to highlight the gaps. Next, we discuss the solution, MONTO_'M'achine-readable 'ONTO'logy_for teaching word problems in mathematics, to fill the gaps. Further, we describe the system implemented using MONTO at the back-end as a proof of concept. This is followed by discussion of results of the study of evaluation of functionalities of the system. Finally, contributions and future work are presented.

\section{State of the art}

Knowledge is related to understanding of the links between different pieces of information. Understanding of a mathematical problem depends on several factors, such as conceptual knowledge, i.e. knowledge of the mathematical facts and concepts and the relations among the facts and concepts, which is also called semantic knowledge (part of the cognitive aspect); contextual knowledge, i.e. knowledge of how things work in real world situations, and especially of story situations, which is also called linguistic knowledge (part of the cognitive aspect); and procedural knowledge, i.e. knowledge of the strategies for solving the problem and the components of the correct procedure, which is also called syntactic or schematic knowledge (part of the meta-cognitive aspect). While studying reading and reasoning skills of math problem solvers, Thomas (1988) also discussed all these types as technical knowledge (linguistic), semantic knowledge, and schematic knowledge. 
Cognitive aspect consists of orientation and organization of knowledge i.e., comprehension of concepts and their structure; ability to analyze the problem information based on the knowledge and ability to chalk out a plan of action based on the analysis. Meta-cognitive aspect consists of knowledge about the procedures and strategies, sub-strategies to solve problems; the ability to make decisions for selection of knowledge component and strategies to solve a problem; the ability to execute the plan of action to solve a problem. Polya $(1946,1981)$ extensively discussed problem types, analysis of problems, and the heuristics for solving a problem. However, one criticism of his model is that it was more focused on cognitive aspects such as understanding the problem and devising the plan, while it ignored the control system, i.e., decision making regarding which strategies must be used for what type of problems. Also, we do not find that Polya $(1946,1981)$ discussed belief systems of students and teachers about mathematics and mathematical problem solving, so another criticism of his model is that it ignored belief systems. Usually research indicates that students find it difficult to categorize the problems and hence to decide which strategy to use. Schonefeld's (1985) framework becomes important as it has addressed both the meta-cognitive aspect and belief systems. While discussing inclusion of cognitive skills in the theory for designing problem-solving learning environments, Jonassen (2011) described requirements of defining problem schema; use of analogy for comparison (which is also mentioned by Polya 1946); understanding causal relationships i.e., semantic and schematic relationships, also mentioned by Schoenfeld (1985) and Maye (1998); question strategies to support problem solving (which is also mentioned by Polya 1946 as a decomposing and recombining strategy); modeling problems; and use of meta-cognitive regulations i.e., meta-comprehension, self-monitoring, meta-cognitive monitoring, self-directed learning, self-regulated learning (which is also mentioned by Polya 1946 as 'looking back'). Meta-cognitive monitoring, i.e., self-regulated learning, can be possible only after modeling of a student. For student modeling, one needs to capture a student's previous knowledge and monitor the ongoing performance for diagnosis of a problem and generation of constructive feedback.

While reviewing the research on teaching and learning of mathematical problem solving, Kilpatrik (1985) mentioned that the question of mastering the model or modeling the master in mathematical problem solving remained unaddressed.

Table 1 List of parameters and detail criteria of the framework

\begin{tabular}{ll}
\hline Parameters & Criteria \\
\hline Knowledge Representation & Linguistic knowledge \\
& Semantic knowledge \\
& Syntactic knowledge \\
Meta-cognitive Aspects & Multiple knowledge representation \\
& Controls/decision-making \\
& Modeling of problem strategies \\
& Questioning to support problem-solving \\
& Modeling of problems \\
& Modeling of students \\
\hline
\end{tabular}


We have developed a framework (see Table 1) consisting of a number of criteria extracted from the literature to evaluate the existing available systems for tutoring mathematical problem solving so that we could find the gaps in the existing systems with respect to the literature. Generally speaking there are two major parameters that are considered: Knowledge Representation and Meta-cognitive Aspect. The criteria for each parameter can be seen in Table 1 .

Some schema models such as ROBUST (Bakman 2007) and CHIPS (Briars and Larking 1984) have also been developed for teaching arithmetic word problems. CHIPS is an integrated model for simple arithmetic word problems and implemented as a system based on production rules. The basic idea of CHIPS was that children naturally understand the problem situation by acting it out either practically or in visualization. Basically CHIPS solved problems about basic actions: giving, losing, finding, and buying. Along with these four actions, CHIPS had control structures named moving and counting. The claim was that it could build a rich problem representation and also supported alternative calculations. Bakman (2007) criticized CHIPS for two reasons: for handling only one change verb (that is, to give) and for not handling the change in format. That means CHIPS could not handle the change of place verbs like to put, to move, and the creation or termination of objects like to build. Also, it could handle a problem only if its sentence described the number of objects the owner had to begin with and the second sentence contained the verbs gave or takes. According to Bakman (2007), the models created for the understanding of word problems had a major limitation: they could represent elementary arithmetic problems but not multi-step complex arithmetic problems. Therefore Bakman (2007) developed a ROBUST schema which could handle multi-step arithmetic problems, problems including all types of verbs and problems with free format. His basic argument was as follows: there is only change schema, and formula is a means to recognize the change of schema instantiations. LIM-G is a learner-initiated model developed by Wong et al. (2007) which is based on cognitive knowledge for understanding and solving geometry word problems. LIM-G proposed to help students in comprehension of geometry word problems. In this, comprehension is achieved by providing multiple knowledge representations of a word problem. Wong et al. (2007) used an ontology-based knowledge engineering tool named InfoMap (Hsu et al. 2001) that includes problem classes, problem concepts, and lexical knowledge. Studies based on Sweller's cognitive load theory found that multiple representations of graphical, textual, and other problems facilitate learning and reduce the cognitive load to integrate the instruction (Sweller and Cooper 1985; Ward and Sweller 1991). Cognitive load is the number of interactive information elements that need to be processed simultaneously before any meaningful learning takes place (Sweller 1988).

There are several systems such as ACTIVEMATH (Melis and Siekmann 2004), Khan Academy (Khan 2004; Sahlman and Kind 2011), WORDMATH (Looi and Tan 1996), Cognitive Tutors (Anderson 1993) (available over the internet either free or on a subscription basis), and LIM-G (Wong et al. 2007) designed for tutoring mathematics in general and word problems too. WORDMATH is a computer-based environment designed and developed by Looi and Tan (1996) for helping students learn and explore word problem solving; it is for simple arithmetic word problems 
with the operations addition and subtraction. In this environment, they used models to visualize things in the word problem so that the student can actually move the models: e.g., the student can use a set of blocks to understand the process of simple arithmetic operations in a real-life context. They claimed that it engages students actively and reflectively in arithmetic word problem solving. Based on Newell's (1990) theory of unified cognition, Anderson et al. (1995) developed a theory named Adaptive Control of Thought Rational (ACT-R) to explain the full range of human cognition. This ACT-R theory has two main parts: the first one is declarative knowledge, which is storage of facts and relationships among the facts, and the second one is procedural knowledge, which is storage of procedures for doing some action. Anderson believed that cognitive skill depends upon conversion of knowledge into production rules, hence together with his colleagues he designed and developed the idea of Cognitive Tutors based on ACT-R theory. This has a knowledge model as part of declarative knowledge and a set of production rules as part of procedural knowledge at the back-end (Anderson et al. 1995). If the student is making a mistake that is not pre-recorded in the knowledge model, it does get highlighted but the student does not get constructive feedback about it: i.e., it does not answer the questions Why did the student go wrong? and What misconception does the student have?. This means that Cognitive Tutors are using cognitive models at the back-end but do not use student models and diagnose each step to provide accurate constructive feedback. If we observe the other systems (such as LIM-G), we find that although these systems offer some kind of diagnosis, they do not create student models and club them with the diagnostic module to provide constructive feedback about where the student went wrong and why. If student models are clubbed with diagnostic module and adaptive component, capturing the student's prior knowledge will be possible and providing a diagnosis of the actual difficulty would be practical, thus providing constructive feedback. ACTIVEMATH (Melis and Siekmann 2004) focuses on high school and college level mathematics and offers an interactive-exploratory learning environment to learners. It uses ontology for knowledge representation as well as for annotation of learning objects (LOs), which contributes to re-usability and interoperability of the content. It also provides various kinds of exercises and a user-friendly environment for practicing exercises. However, there is no evidence found regarding the use of analytical questions to facilitate understanding of how to solve a problem; in particular, word problems are not handled in ACTIVEMATH. Khan Academy is a very good example of a distributed learning environment. It began with a collection of small videos for teaching basic concepts of mathematics and science, and later on developed into a blended learning environment for mathematics. The videos are arranged on the basis of a knowledge map that resembles the notion of concept map.

Concept maps are visual depictions of relationships between various concepts and are graphical tools for the structuring and representation of knowledge (Novak and Canas 2008). Students can explore the concepts through the knowledge map, which gives them an idea of how various concepts are interconnected. Students can watch the video, and after learning that concept they can solve the exercises. Students' interaction with the system gets caught by the system, and an advanced learning profile named accomplishments is presented to the student. In the Indian 
context, the American English accent and the American context of the examples given while explaining a concept are possible problems with the Khan Academy. In Fig. 1, the availability of a criterion for a system is shown by a cross mark and unavailability is shown by a blank. The gray textured boxes with cross mark show that the criterion exists but is explored in a weak manner. From the literature review and evaluation of the systems against the parametric framework (refer to Fig. 1) it is concluded that modeling of problem strategies, asking analytical questions to students, building student models for diagnosis of students' missing concepts (a subset of all concepts which are expected to be known to experts but are not known by students) and misconceptions (these are created by students on their own due to misunderstanding) offering remedial feedback is less researched. For teaching word problems, semantic and syntactic knowledge representation is useful together with multiple knowledge representations for reducing cognitive load and better understanding.

\section{The solution: MONTO}

Ontology is a specification of a conceptualization (Gruber 1993). Motivated by the arguments given in earlier sections, we now present an ontology, named MONTO, for teaching word problem solving in mathematics. The proposed MONTO

\begin{tabular}{|c|c|c|c|c|c|c|c|}
\hline $\begin{array}{c}\text { Parameters } \\
\& \text { Criteria }\end{array}$ & $\begin{array}{l}\text { LIM-G } \\
(2005)\end{array}$ & $\begin{array}{l}\text { WORD } \\
\text { MATH } \\
(1996)\end{array}$ & $\begin{array}{l}\text { Cognitive } \\
\text { Tutors } \\
\text { (1995) }\end{array}$ & $\begin{array}{c}\text { ROBUST } \\
(2007)\end{array}$ & $\begin{array}{l}\text { CHIPS } \\
(1984)\end{array}$ & $\begin{array}{c}\text { ActiveMath } \\
(2004)\end{array}$ & $\begin{array}{c}\text { Khan Academy } \\
\text { (2011) }\end{array}$ \\
\hline $\begin{array}{c}\text { Linguistic } \\
\mathrm{KR}\end{array}$ & $x$ & & $x$ & & $x$ & & \\
\hline Semantic KR & $x$ & & $x$ & $x$ & $x$ & $x$ & $x$ \\
\hline Syntactic KR & $x$ & & $x$ & $x$ & $x$ & & \\
\hline Multiple KR & $x$ & $x$ & $x$ & & & $x$ & \\
\hline Controls & $x$ & & $x$ & & & $x$ & $x$ \\
\hline $\begin{array}{l}\text { Modeling of } \\
\text { Problem } \\
\text { Strategies }\end{array}$ & & & $\bar{x}$ & $x$ & $x$ & & $\bar{x}$ \\
\hline Questioning & $x$ & & $\bar{x}$ & & & & \\
\hline $\begin{array}{l}\text { Modeling of } \\
\text { Problems }\end{array}$ & $x$ & $x$ & $x$ & $x$ & $x$ & $x$ & \\
\hline $\begin{array}{l}\text { Modeling of } \\
\text { Students }\end{array}$ & & & $x$ & & & $\bar{x}$ & $x$ \\
\hline
\end{tabular}

Fig. 1 Evaluation of the systems against the parametric framework 
ontology is divided into four main parts: system/pedagogy ontology, strategy/task ontology, user model/student ontology, and domain ontology. For solving problems, a student must know the concepts and the relations between the concepts used in that particular problem. Figure 2 shows a screenshot of general classes in the MONTO.

The domain ontology means all the conceptual connections needed by students, which are described as resources in the framework for mathematical thinking discussed by Schoenfeld (1992). To solve any problem, the student also needs to know various problem-solving strategies that can be used to solve the problem. The ontology of problem-solving strategies and tasks (strategy/task ontology) needed to be done by students is described in the framework as heuristics. Students need to know when to use which concept and strategy to solve any particular problem. Thus, the rules used to manipulate the domain ontology and task ontology can be described as control components in the framework. The fourth component, belief system, is an implicit factor or effect of the other three components, and hence would not be considered in the ontological structure. Thus, the notion of use of ontology fits very well into the first three components of the framework for mathematical thinking given by Schoenfeld (1992). One can even use an ontological structure to describe the connection between linguistic terms and mathematical notions used in the real word and story problems. Figure 3 shows a screenshot of Problem Schema class and its instances, from which the schematic, semantic, and linguistic knowledge is extracted. Schematic knowledge is extracted from the names of the sub-classes of the Problem Schema class with respect to that particular problem. The semantic and linguistic knowledge is extracted from the instance connection of the domain knowledge named hasFeature and the class of that instance connection from the domain knowledge. For example, in Fig. 3, the problem schema for CoveringProblem is shown. Height_Of_Conical_Heap_Of_ Wheat is the linguistic knowledge in connection with Height of a right circular cone, which is the semantic connection of the schematic knowledge component Dimension_Of_Object_To_Be_Covered.

System/pedagogy ontology can also be used for teaching problem solving together with domain and task ontologies, which is described in "The ontologybased system" section. Figure 4 shows a screenshot of system/pedagogy ontology classes in MONTO in which analytical questions are modeled as per the problem type as instances of the sub-classes of the Question class. Each question has a set of answers that are modeled as a sub-class of the Answer class. The Problem class is connected with the Question class and the Question class is connected with the Answer class. The Answer class is connected with the Feedback class. Each incorrect answer has a remedial feedback and also connects to the Bug class. Figure 5 shows a screen shot of Bug class in MONTO from which students' missing concepts and misconceptions are modeled and connected to other classes in the ontology. These are used in student modeling to show the missing concepts or misconceptions of a student and provide remedial feedback. Missing concepts are a sub-set of domain knowledge, but misconceptions can be anything that is developed by students on their own. 
Fig. 4 Screenshot of system/ pedagogy ontology classes in MONTO



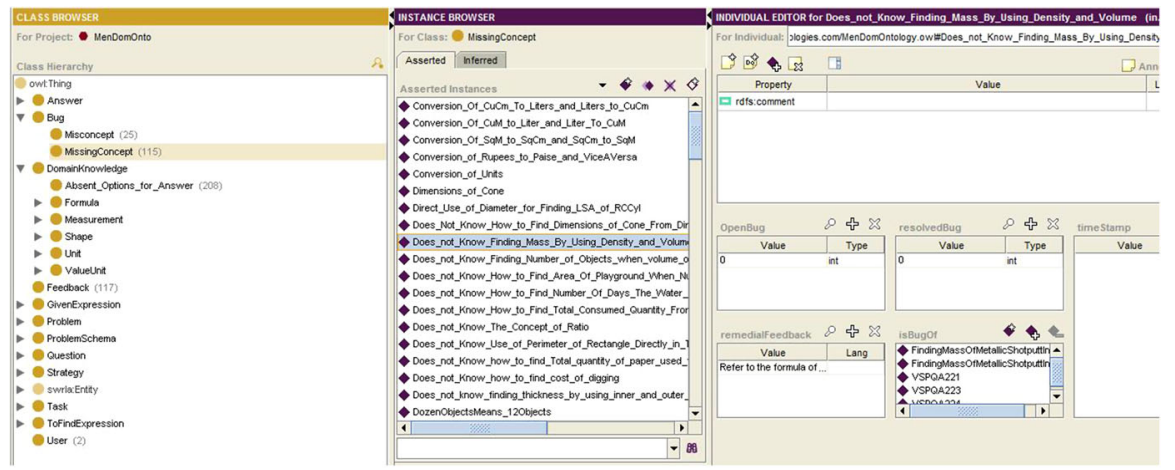

Fig. 5 Screenshot of Bug class in MONTO

\section{The ontology-based system}

This section describes a system developed based on the MONTO ontology. This ontology-based system has three major components: Learning Mode, Solving Mode, and Student's Learning Profile. After a student logs onto the system using User Name and Password, the system displays the list of problem types from which the student can select a problem type and the difficulty level, i.e., easy, medium, or hard. Every word problem contains some real world scenario together with some 


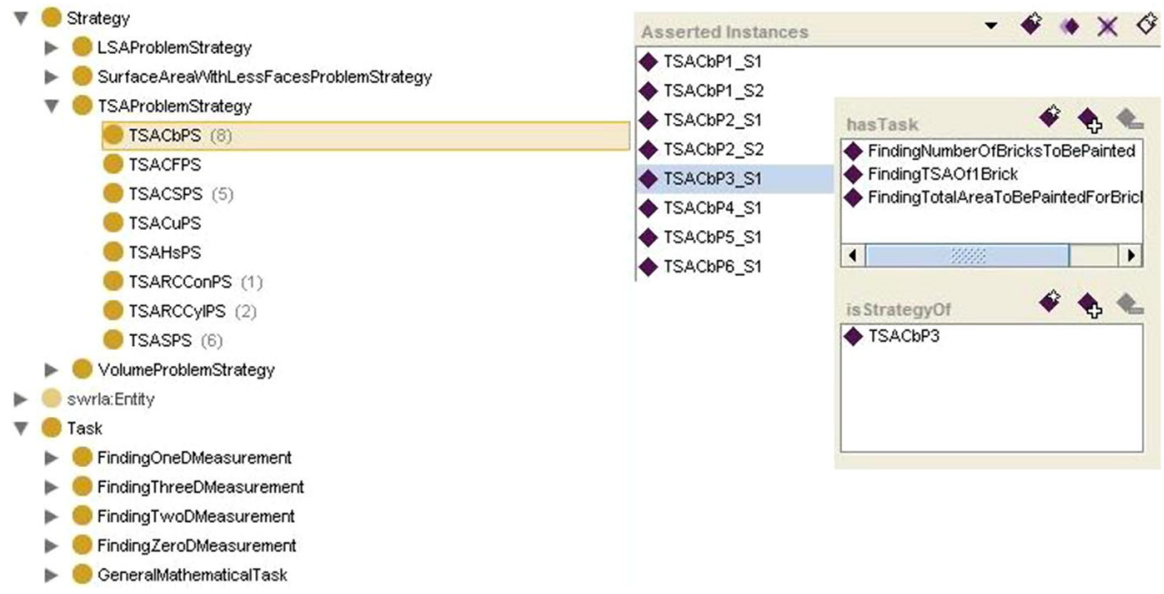

Fig. 6 Screenshot of strategy/task ontology in MONTO

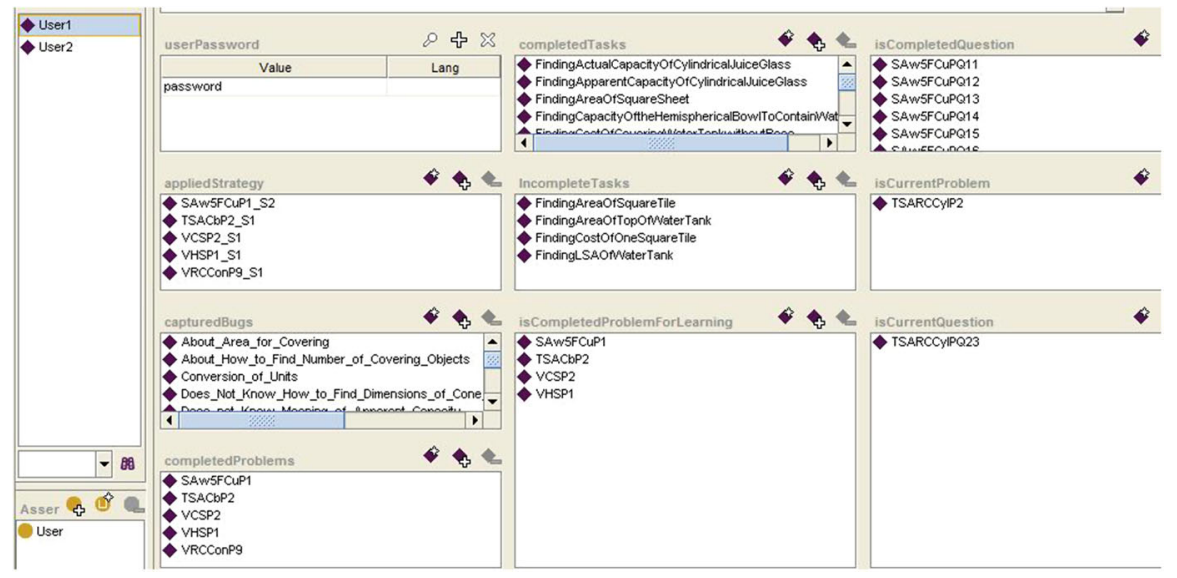

Fig. 7 Screenshot of student model ontology in MONTO

given items (Given) and some items that need to be found (To-find). A problem in which the Given and To-find items are given directly is considered an easy problem. A problem whose schema contains a greater number of Given items and has some complex real world scenario is considered a medium problem. A problem whose schema contains a greater number of items in which the Given and To-find items are given indirectly and a problem that has a more complex real world scenario is considered a hard problem. Figure 8 shows a screenshot of a problem modeled in MONTO. Each problem has a difficulty level, a problem schema, a set of analytical questions, a set of answers and remedial feedback, a set of given expressions and tofind expressions, and strategies for solving the problem. 
Once a problem type and the difficulty level are selected, the system displays a problem statement and two options: Learn How to Solve and Solve the Problem. If a student selects Learn How to Solve, the system takes the student to Learning Mode and if the student selects Solve the Problem, the system takes her to solving mode. Learning Mode offers a tutoring module where the system asks the student analytical questions that can help her to analyze a problem (see Fig. 9). This provides training in how to think in order to solve any given problem. Solving mode has various parts, such as Schematic Knowledge, Semantic Knowledge, Formula, Image, Given, To-Find, and Solution. Please refer to Fig. 10 that shows a screenshot displaying solving mode of the system in which one can see Schematic and Semantic knowledge displayed in the system. Learning mode helps a student analyze a problem and devise a plan to solve the problem by asking analytical questions, offering answer choices and providing constructive feedback. On the other hand, solving mode enables her to solve the problem on her own. The student can enter given items in the problem in the text area for Given and To-Find items in the text area of To-Find. Also, student can enter the tasks step by step in the text area of Solution.

All the interactions of the student with the system are stored back in the student model ontology in MONTO (see Fig. 7). The information captured by the student model ontology is used for displaying the student's learning profile (see Fig. 11). There are six parts of the student's learning profile: (a) Problem-Solving Progress; (b) Misconceptions; (c) Missing Concepts; (d) Learned Concepts (specific to a particular problem type); (e) Learning profile of Mensuration Domain; and (f) Activity. When the student clicks on the tab Student's Learning Profile, the list of six parts is displayed. Problem-Solving Progress is for all problem types in general; in it, a student can see how many problems of each level she has solved for each problem type and the percentage of problems that she has answered correctly. Also, it combines the percentage of correctness of all the three levels and shows overall percentage of correctness. This information can be used by both the student

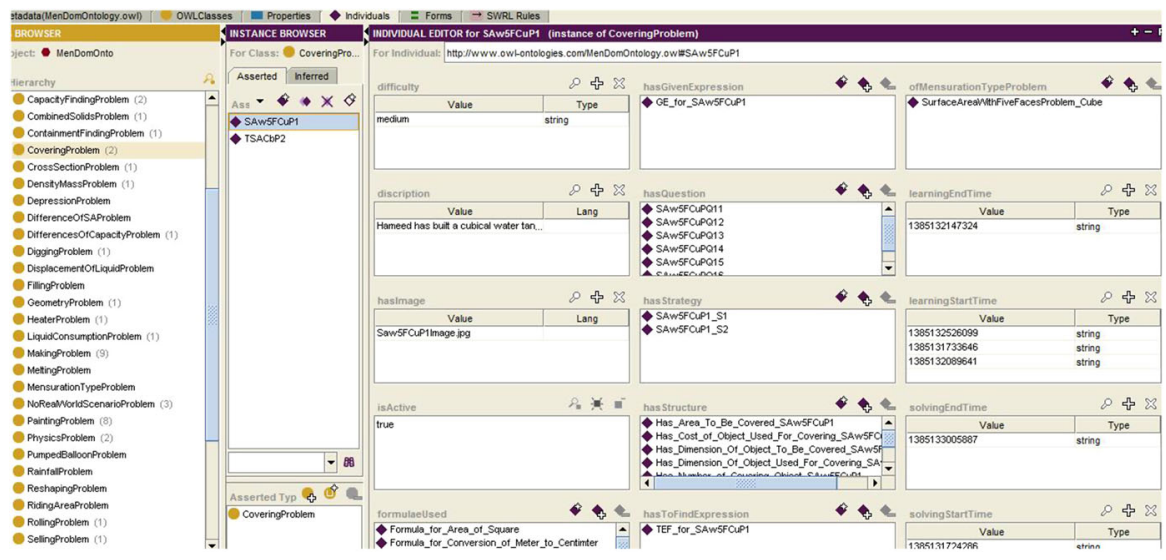

Fig. 8 Screenshot of a problem modeled in MONTO 


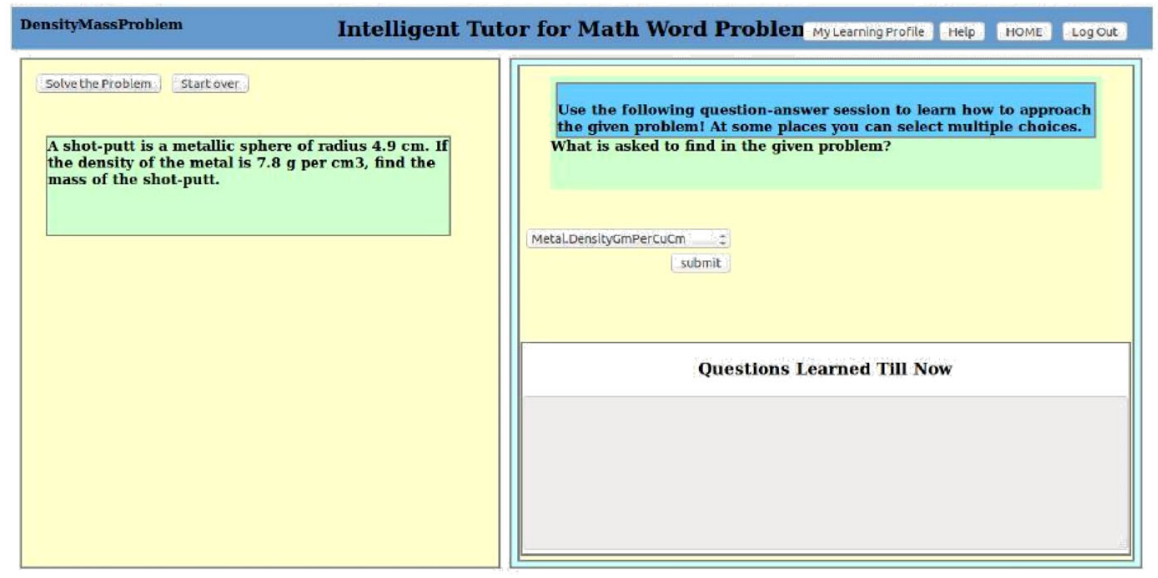

Fig. 9 Learning mode screenshot

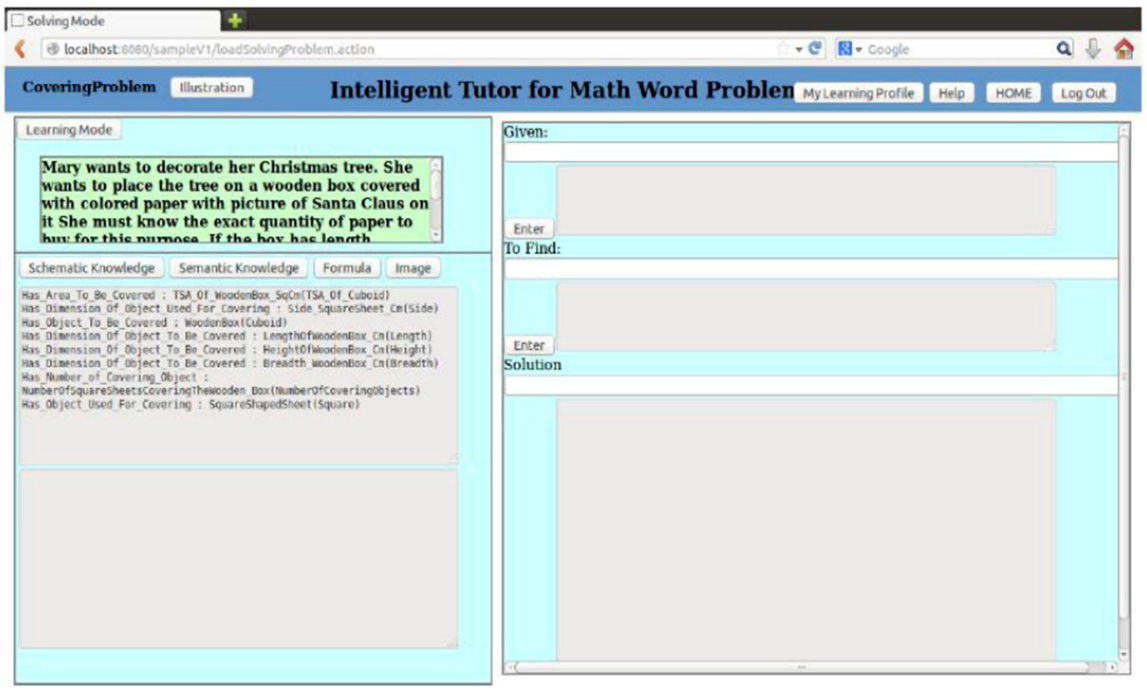

Fig. 10 Solving mode screenshot

and the teacher to find out how much learning of specific problem types has taken place and what difficulty levels have been covered, so that the student can decide how and what to study further and the teacher can decide what to focus on while teaching or revising. When a student interacts with the system in Learning Mode and Solving Mode, the student model captures the information about how many tasks the student has completed, how many tasks the student has not completed, how many questions the student has answered correctly, how many times the student has answered a question incorrectly, etc. These Missing Concepts and Misconceptions 


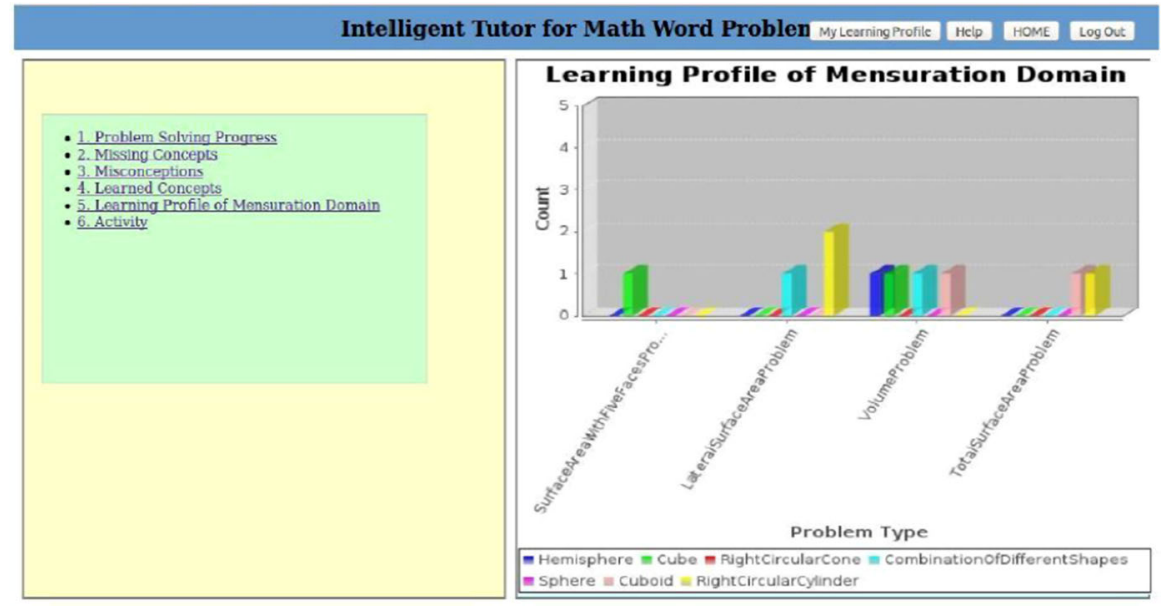

Fig. 11 Student's learning profile screenshot

are not problem-specific but domain-specific, as many problems may demonstrate the same missing concept or misconception. Together with a list of missing concepts and misconceptions, the percentage of times the bug is resolved is shown along with remedial feedback. This remedial feedback may contain a link to another resource to study the concept in depth. Also, by conducting more analytics one can find common missing concepts and misconceptions of a group of students and can tailor the teaching methodology as per the need. Also, this information serves the diagnostic function of identifying the missing concepts and misconceptions of a particular student or even a class of students. Learned Concepts displays the information about concepts a student has learned for a particular problem type in a tabular form that gives a list of concepts and the number of times the student has learned each concept. With the displayed learned concept, one can see in the brackets the schematic connection of that particular problem type. Also, the same is shown using 3D Bar charts. Learning profile of Mensuration Domain shows information about the mensuration problem types a student has learned for each shape. For example, it displays the percentage of TSA problems learned for each shape, the percentage of LSA problems learned for each shape, the percentage of SA with Less Faces problems learned for each shape, and the percentage of volume problems learned for each shape. The purpose of this is to get the knowledge about the shapes and their respective measurement type problems directly from the ontology. So if we edit the ontology, then the learning profile domain is expected to change according to the edits. In Fig. 11, a screenshot of the fifth part (Learning profile of Mensuration Domain) is shown. Activity shows how much time (in seconds) a student has spent on Learning Mode and Solving Mode for various problem types. 


\section{System and component architecture}

The system can be broadly divided into four modules: user interface, logic, data access, and ontology file. User interface is a collection of Java Server Pages (JSP) files. Logic module is the level below user interface. It receives input from the user interface and translates it into function calls. The function calls access data access modules or functions within the logic module. The logic module gets the result from the data access module after processing and displays it in the user interface. The logic module acts as the only interface between the user interface and the data access module. Some functions of logic module are getFirstQuestion(), validateGiven(), checkAnswer(), loadLearningMode(), etc. Data access module has lower level functions that work directly with the Web Ontology Language (OWL) file and the Relational triple store. The logic module calls corresponding functions from the data access module. The data access module translates them into data level functions and returns relevant data structures. Some data access functions are runQuery(), loadData(), ndTriple(), insertProperty(), etc. The data needed by the system is stored in a Relational Database in the form of triples.

An OWL file is an ontology file stored in RDF format. Because it is a flat file, direct querying of the file resulted in poor system response time; hence the ontology is converted into RDBMS triple store using SDB framework of JENA API. The OWL file consists of Classes, Objects, and Properties. Properties are used to connect objects to other objects or objects to data types. The ontology file also consists of rules in SWRL format. Please refer to Fig. 12. The class files are present in the logic module and Data Access (Data structures) module. The Logic module classes interact either directly with RDF store using SPARQL or through Data module

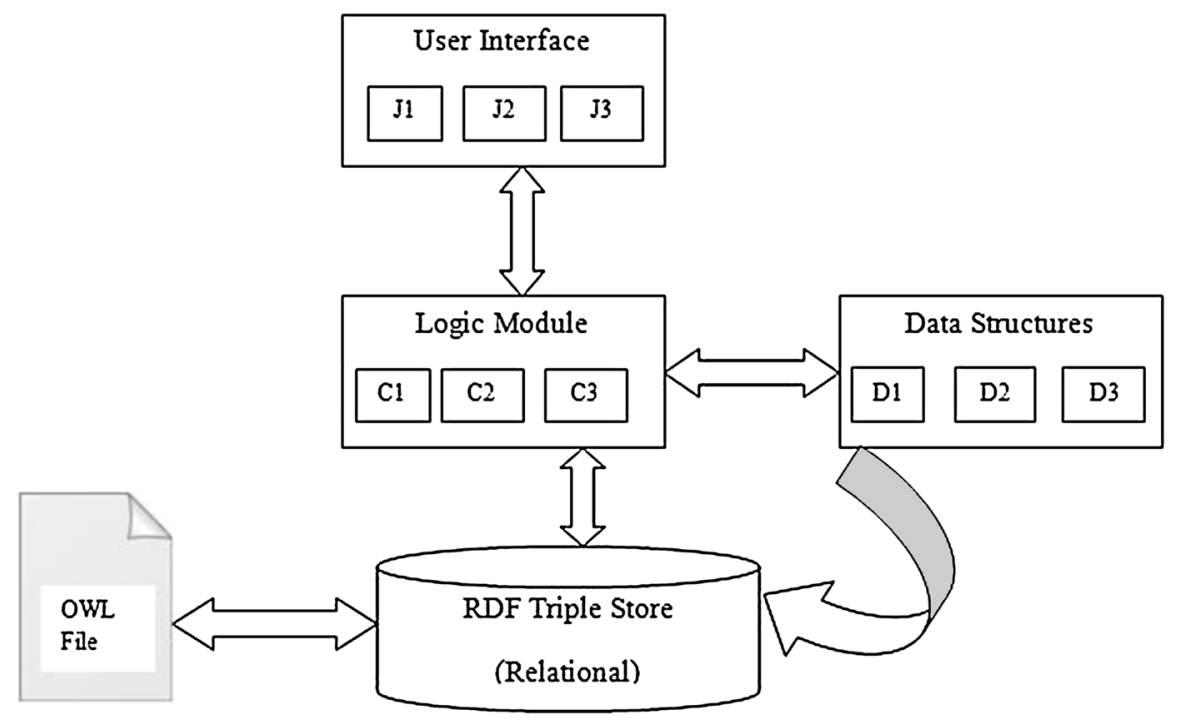

Fig. 12 System architecture 
classes. The RDF relational store is updated from the OWL file using SDB framework of Jena. This is a one-time process done using a script. All updates to the ontology are done in the relational RDF store which updates to the OWL file on specific events like Completion of a problem in solving mode or during logout. The advantage of keeping the triple store in a relational database is much faster response time while fetching the data from the ontology when compared to data retrievals from a flat file. The logic module is divided into multiple files developed using the Java programming language. Each Java file has an associated JSP file. The interaction between various Java files and JSP files is described in Fig. 13. The data access module consists of four Java files: GlobalData.java, Tables.java, OwlAccess.java, and RDFAccess.java. OwlAccess.java contains all functions that interact directly with the ontology file. RDFAccess.java contains all functions that interact with the RDF store. Both OWLAccess and RDFAccess are singleton classes. GlobalData contains data needed for learning mode and Tables contain all data needed for solving mode. Please refer to Fig. 13. The system is implemented as a multi-tier architecture. Such an architecture increases the independence of each module. In our system, the ontology is continuously evolving. An evolving ontology means continuous change in data format, data structures, etc. We need a system that is open to change. A loosely tied multi-tier system fits these needs. The level of

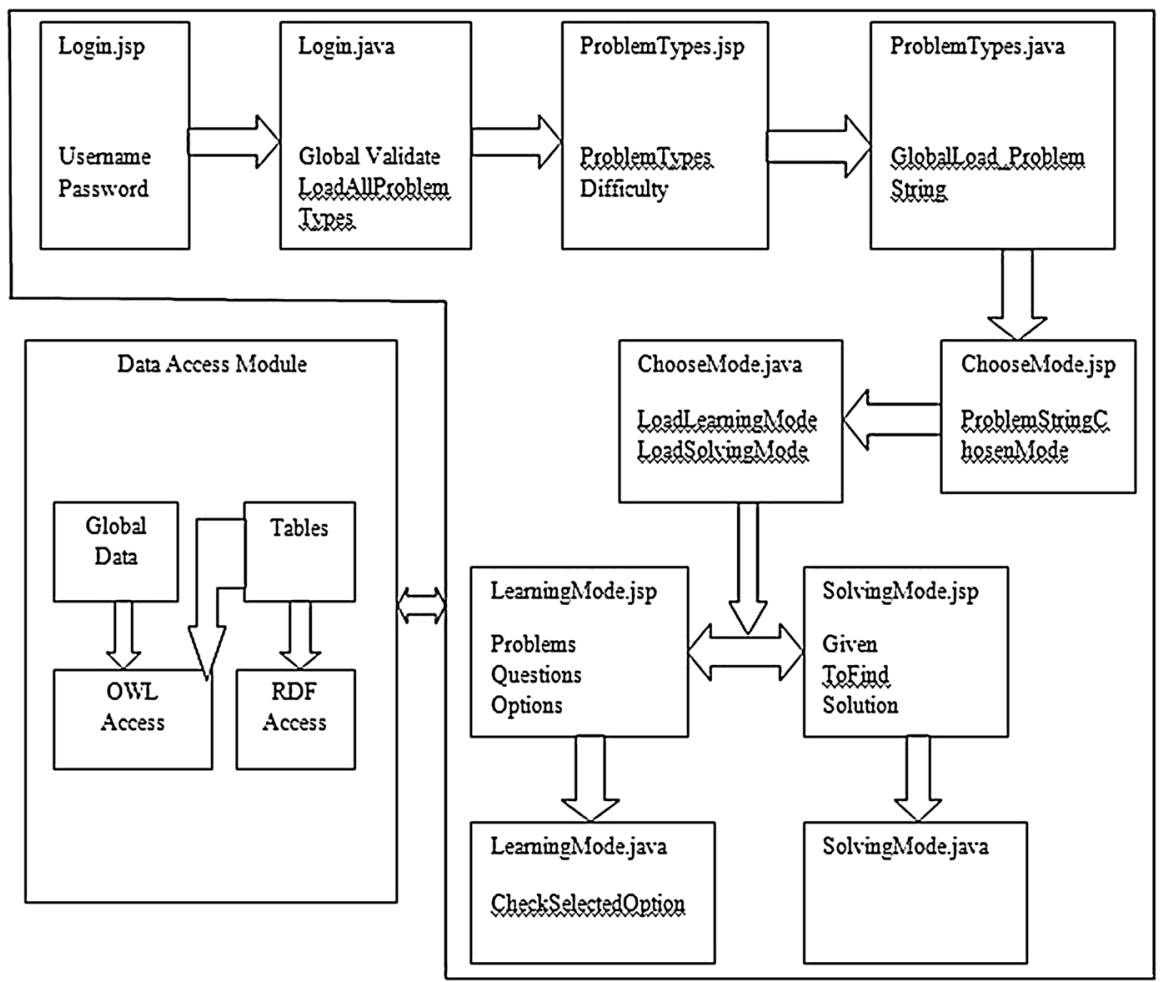

Fig. 13 Component architecture 
independence is not absolute due to query result format of SPARQL (SPARQL Protocol and RDF Query Language).

\section{The functionalities derived from ontology}

Smart learning environment is an environment that uses self-monitoring, analysis, and reporting technology. Without pedagogy and expert knowledge one cannot develop a self-learning environment. Self-monitoring, analysis, and reporting can be done using the data captured in the student model and using learning analytics. Following are the functionalities of the system that are derived from the ontology used at the back-end: (a) Ontology is used in all the four parts (pedagogy module, expert module, problem-solving module, and student module) of the smart learning environment. Pedagogy ontology is used in tutoring module, domain ontology is used in expert module, task ontology is used in interface module or problem-solving environment, and user model ontology is used in student module. (b) The list of problem types and the list of difficulty levels from which a student selects the type of problem and the difficulty level are directly fetched from the ontology. (c) In learning mode, the analytical questions asked and the choices offered by the system are derived from ontology. (d) The constructive feedback provided for each action (i.e., selection of correct or incorrect answer) is also taken from the ontology. (e) In solving mode, the correct items that are accepted into the text areas of "Given" and "To-find" are fetched from the ontology. (f) The correct items that are accepted into

\begin{tabular}{|c|c|c|c|c|c|c|c|c|}
\hline $\begin{array}{l}\text { Parameters } \\
\text { \& Criteria }\end{array}$ & $\begin{array}{l}\text { LIM-G } \\
(2005)\end{array}$ & $\begin{array}{l}\text { WORD } \\
\text { MATH } \\
\text { (1996) }\end{array}$ & $\begin{array}{l}\text { Cognitive } \\
\text { Tutors } \\
\text { (1995) }\end{array}$ & $\begin{array}{l}\text { ROBUST } \\
\text { (2007) }\end{array}$ & $\begin{array}{l}\text { CHIPS } \\
\text { (1984) }\end{array}$ & $\begin{array}{c}\text { ActiveMath } \\
\text { (2004) }\end{array}$ & $\begin{array}{l}\text { Khan Academy } \\
\text { (2011) }\end{array}$ & $\begin{array}{l}\text { MONTO Based } \\
\text { System (2014) }\end{array}$ \\
\hline $\begin{array}{l}\text { Linguistic } \\
\mathrm{KR}\end{array}$ & $x$ & & $x$ & & $x$ & & & $x$ \\
\hline Semantic KR & $x$ & & $x$ & $x$ & $x$ & $x$ & $x$ & $x$ \\
\hline Syntactic KR & $x$ & & $x$ & $x$ & $x$ & & & $x$ \\
\hline Multiple KR & $x$ & $x$ & $x$ & & & $x$ & & $\begin{array}{c}\mathrm{X} \\
\text { (Implementation } \\
\text { Specific) }\end{array}$ \\
\hline Controls & $x$ & & $x$ & & & $x$ & $x$ & $x$ \\
\hline $\begin{array}{l}\text { Modeling of } \\
\text { Problem } \\
\text { Strategies }\end{array}$ & & & $x$ & $x$ & $x$ & & $x$ & $\mathbf{x}$ \\
\hline Questioning & $x$ & & $\mathrm{x}$ & & & & & $x$ \\
\hline $\begin{array}{l}\text { Modeling of } \\
\text { Problems }\end{array}$ & $x$ & $x$ & $x$ & $x$ & $x$ & $x$ & & $x$ \\
\hline $\begin{array}{l}\text { Modeling of } \\
\text { Students }\end{array}$ & & & $\bar{x}$ & & & $x$ & $x$ & $\begin{array}{l}\mathrm{X} \text { (Missing and } \\
\text { Misconceptions) }\end{array}$ \\
\hline
\end{tabular}

Fig. 14 MONTO filling gaps in the evaluation rubric (Lalingkar et al. 2015) 
the text area of "Solve" are fetched from the various tasks listed under the Task class for that particular problem in the ontology. (g) Schematic knowledge, semantic knowledge, list of formulas, and a static image for a particular problem are also derived from the ontology at the back-end. (h) The information displayed in a student's learning profile-namely Problem-Solving Progress, capturing of bugs (i.e., missing concepts and misconceptions), the remedial feedback provided for each bug, Learned Concepts, the learning profile of the mensuration domain, and the activity-is also derived from the ontology used at the back-end. Thus, the ontology plays a central role in the system for addressing several limitations of other systems in teaching mathematical problem solving.

\section{Comparison of MONTO-based system with the existing systems}

After reviewing the literature about the teaching of mathematical problem solving, it has been found that a ROBUST schema has been developed by Bakman (2007) for understanding arithmetic word problems. This schema is static, and also has not been implemented as a tutoring system. MONTO used at the back-end can be reused for many problems and can be interoperable. In LIM-G, the student model is not incorporated; in Cognitive Tutors the student model is incorporated, but in a somewhat weak manner. This is discussed in "State of the art" section. MONTO addresses the problem of communicating to a student where she is wrong, why she is wrong, and what she should do to rectify that mistake. The MONTO-based system uses Bug class and Feedback class in MONTO to convey this information to the student. In ActiveMath and Khan Academy, there is no evidence of asking analytical questions to students, and in MONTO analytical questioning is modeled along with remedial feedback. Also, MONTO models the problem strategies and provides more granularities of the mistakes in the form of missing concepts and misconceptions, which is not evident in most of the systems. Figure 14 shows the way the MONTO-based system fills the gaps identified by the review of existing systems in the state of the art; the gaps are shown in Fig. 1 and discussed in "State of the art" section.

\section{Limitations of the implementation of the system}

Following are the limitations of the implementation of the system, making it not yet ready for students' use.

1. Some rigidity has been enforced in the format for filling the text in Solving Mode:

(a) Only parentheses are allowed for sequencing of operations in a formula expression. Symbols like [] are not allowed.

(b) The space between Value and Unit is mandatory. 


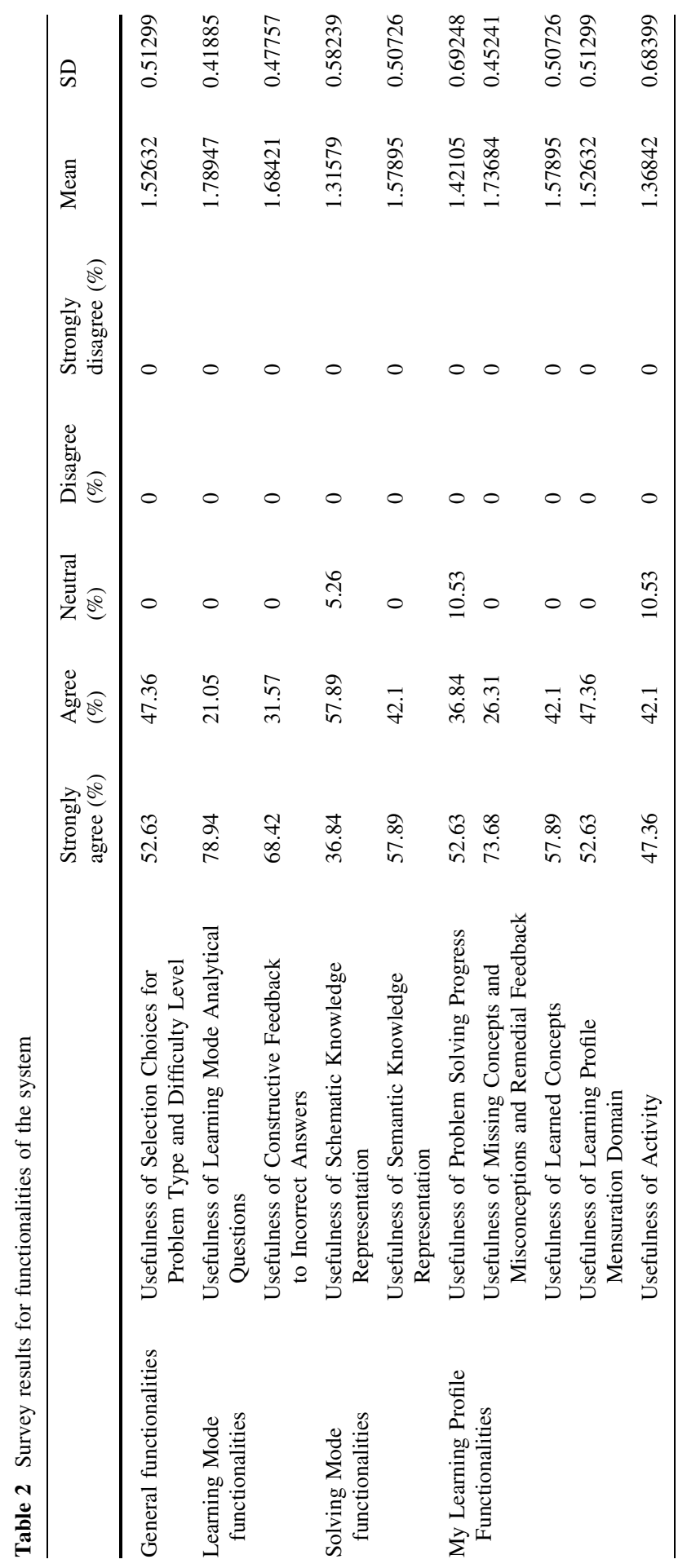


(c) The object name and even the measurement name have to be written in a specific format; for instance, Total Surface Area of Cuboid in Square Centimeter can only be written in the format Cuboid.TSASqCm

2. There is some delay in the performance after completion of solving mode or after logout, since the list of triples gets copied from RDF store to OWL file.

3. A few design issues (such as the fact that the inverse property between question and problem is not used) have made the query execution slow in a few cases.

4. The unit conversions are taken from the static text file instead of from the ontology; this leads to irregularities while deploying the application to another system.

5. The diagram tool is not implemented for showing the diagrammatic representation.

6. Some bugs exist in the program, such as the following: the student is permitted to start the solving mode with the solution part even when the "given" and "tofind" parts are empty.

Most of these limitations can be rectified by better design and improvement of the code as well as some improvements in the ontology.

\section{Evaluation of the functionalities}

The focus and contribution of this study are the functionalities of a smart learning environment that are derived from MONTO - which fills the gaps identified in the evaluation rubric - and not the implementation of the fully working system and its effectiveness. Hence, we evaluated the usefulness of the functionalities of the system, which are drawn from ontology, with the help of interactions with schoolteachers. A set of structured questions based on a five-point Likert-like scale were used. The questions were based on the usefulness of the functionalities of the system that are derived from the ontology. Teachers were oriented about the ontology and the system and then were asked to interact with the system. During or after the interaction, they were asked to answer the survey questions. Results are shown in Table 2.

A total of 19 teachers of mixed Information Technology (IT) proficiency responded and participated in the survey. All of them were mathematics teachers but at various levels. A majority of the teachers taught mathematics at high school level. It is observed that more than $95 \%$ of the teachers have strongly agreed or agreed to the assertive statements about the usefulness of the system's functionalities. After finishing the structured opinionnaire, the teachers were also asked to give their opinions about the system in free-form text. One teacher said, "After seeing the way questions are asked in analytical mode and reading the distractors or even the constructive feedback, I learned many things which I had never thought of in my twenty years' service. I am interested in knowing about how to create ontology." Some of the teachers said that they had seen many educational softwares sold in the 
market, but had not seen the same level of granularity of captured mistakes that is seen in this system. When asked to elaborate, they talked about the diagnosis of missing concepts and misconceptions. This opinion can be seen reflected in the results of the structured opinionnaire. A few other teachers said that they could not quite visualize the schema of a problem and were not convinced by the schematic knowledge representation. Hence, they could not give an opinion.

\section{Summary and conclusions}

In summary, the MONTO ontology fits very well into the general architecture of the Intelligent Tutoring Systems (pedagogy ontology to pedagogy module, Domain Ontology to expert module, task ontology to problem-solving environment, and student model ontology to student module) that can be considered as part of a smart learning environment. The MONTO ontology at the back-end is used for eight critical aspects (mentioned in "The functionalities derived from ontology" section) of the smart learning environment. It is concluded that the use of the MONTO-based system bridges the gaps highlighted in the state of the art study (See Fig. 14). A more effective implementation (constraint-free text entry, different way of presentation of schematic and semantic knowledge, inclusion of diagrammatic tool for drawing diagrams) would make the system more user-friendly. This ontology can also be used for drawing diagrams automatically for a given problem by using the problem schema. Those diagrams can be matched with the diagrams drawn by students to find missing concepts and misconceptions of the students. Using natural language processing (NLP) and machine learning (ML) techniques, one can generate problems similar to the problems being modeled in the ontology and create analytical questions for a given word problem that can be used in the pedagogy ontology. A completely functional system could use ML techniques to learn how problems are solved by good students and the same strategies could be taught to weak students. MONTO can be used as a part of a bigger adaptive smart learning environment to teach mathematics. With student profiles captured in MONTO, many more learning analytics can be generated for individual students and groups of students.

\section{References}

Anderson, J.R. (1993). Rules of the Mind. NJ: Lawrence Erlbaum Associates, Hillsdale.

Anderson, J. R., Corbett, A. T., Koedinger, K. R., \& Pelletier, R. (1995). Cognitive tutors: Lessons learned. The Journal of the Learning Sciences, 4(2), 167-207.

Bakman, Y. (2007). Robust understanding of word problems with extraneous information. arXiv preprint math/0701393.

Briars, D. J., \& Larking, J. H. (1984). An integrated model of skill in solving elementary word problems. Cognition \& Instruction, 1(3), 245-296.

Gruber, T. R. (1993). A translation approach to portable ontologies. Known Acquisition, 5(2), 199-220.

Hsu, W. L., Wu, S. H., \& Chen, Y. S. (2001). Event identification based on the information mapINFOMAP. In Proceedings of the 2001 IEEE systems, man, and cybernetics conference (pp. 1661-1672), Tucson, Arizona. 
Jonassen, D. H. (2011). Learning to solve problems: A handbook for designing problem-solving learning environments. Routledge: Taylor and Francis Group.

Khan, S. (2004). The Khan academy. Boston: Harvard Business.

Kilpatrik, J. (1985). A retrospective account of the past twenty-five years of research on teaching mathematical problem solving. In E. A. Silver (Ed.), Teaching and learning mathematical problem solving: Multiple research perspectives (pp. 1-15). Hillsdale, NJ: Lawrence Erlbaum Associates.

Lalingkar, A., Chandrashekar, R., \& Ramani, S. (2015). MONTO-A machine-readable ontology for teaching word problems in mathematics. Journal of Educational Technology and Society, under review (submitted).

Looi, C. K., \& Tan, B. T. (1996). Wordmath: A computer based environment for learning word problem solving. Computer Aided Learning and Instruction in Science \& Engineering, 1108, 78-86.

Marshall, S. A. (1995). Schemas in problem solving. Cambridge: Cambridge University Press.

Mayer, R. (1998). Cognitive, metacognitive, and motivational aspects of problem solving. Instructional Science, 26, 49-63.

Melis, E., \& Siekmann, J. (2004). Activemath: An intelligent tutoring system for mathematics. In Artificial intelligence and soft computing-ICAISC 2004 (pp. 91-101). Berlin: Springer.

Newell, A. (1990). Unified theories of cognition. Cambridge: Harvard University Press.

Newell, A., \& Simon, H. A. (1972). Human problem solving (Vol. 104(9)). Englewood Cliffs, NJ: Prentice-Hall.

Novak, J., \& Canas, A. J. (2008). The theory underlying concept maps and how to construct and use them (p. 284). Florida Institute for Human and Machine Cognition Pensacola, FL. http://cmap.ihmc.us/ Publications/ResearchPapers/TheoryCmaps/TheoryUnderlyingConceptMaps.htm.

Polya, G. (1946). How to solve it: A new aspect of mathematical method. Princeton: Princeton University Press.

Polya, G. (1981). Mathematical discovery: On understanding, learning and teaching problem solving. New York: Wiley.

Sahlman, W. A., \& Kind, L. (2011). Khan academy. Boston: Harvard Business.

Schoenfeld, A. H. (1985). Mathematical problem solving. New York: Academic Press.

Schoenfeld, A. H. (1992). Learning to think mathematically: Problem solving, metacognition and sense making in mathematics. In D. Grouws (Ed.), Handbook for research on mathematics teaching and learning (pp. 334-370). New York: MacMillan.

Silver, E. A. (1985). Teaching and learning mathematical problem solving: Multiple research perspectives. New Jersey: Lawrence Erlbaum Associates.

Sweller, J. (1988). Cognitive load during problem solving: Effects on learning. Cognitive Science, 12, 257-285.

Sweller, J., \& Cooper, G. (1985). The use of worked examples as a substitute for problem solving in learning algebra. Cognition \& Instruction, 2, 59-89.

Thomas, D. (1988). Reading and reasoning skills for math problem solvers. Journal of Adolescent \& Adult Literacy, 32(3), 244-249.

Ward, M., \& Sweller, J. (1991). Cognitive load theory and the format of instruction. Cognition \& Instruction, 8, 293-332.

Wong, W., Hsu, S., Wu, S., Lee, C., \& Hsu, W. (2007). LIMG: Learner-initiating instruction model based on cognitive knowledge for geometry word problem comprehension. Computers \& Education, 48(4), 582-601.

Aparna Lalingkar is a full time Ph.D. Student in International Institute of Information Technology, Bangalore. Her research interests are use of technology for teaching problem solving in mathematics, AI applications to Education, use of ICT, and Semantic Web Technology for Mathematics Education. Her primary work is in application of ontology for teaching word problems in Mathematics. She has done her MPhil from Cambridge University, UK in Mathematics Education research and MSc Mathematics from University of Pune. She has wide teaching experience right from tribal students, gifted students to University students. Aparna has received fellowships from HP Labs, India for her PhD and from Cambridge Commonwealth Trust-DFID, UK for her M.Phil. She is serving as member of technical program committee for several conferences in the field. 
Chandrashekar Ramnathan is working as Associate Professor at International Institute of Information Technology (IIIT), Bangalore. He received his Ph.D. degree from Mississippi State University, USA. His thesis was in the area of object-oriented databases. He moved to academia after spending more than 10 years in the software industry. He has extensive application software development experience in large multinational organizations. His current focus is in the area of data sciences and technology for education. Model-based software engineering and enterprise content management are his other areas of interest. He serves on technical advisory panels of the Government of Karnataka and educational institutions. He is also on the Board of BITES (Board for IT Education Standards).

Srinivasan Ramani has served as the founding director of the National Centre for Software Technology. $\mathrm{He}$ was inducted into the Internet Hall of Fame for his contributions to bringing the Internet to India. He has served as the first Director of HP Labs India and as Professor, International Institute of Information Technology, Bangalore. 